\title{
Beware of Neglect of Non-COVID Patients in COVID Era
}

\author{
Anuj M Clerk
}

Indian Journal of Critical Care Medicine (2021): 10.5005/jp-journals-10071-23940

Coronavirus disease (COVID) has created an unprecedented crisis in the field of medicine where it blew a double blow on population. All news channels covered amply the sufferings of COVID patients. Daily case rates and death rates draw much-needed attention at national and international, but it overshadowed the sufferings of other substantial and larger segments of patients, "The non-COVID patients."

This occurred in three ways: (1) The majority of hospitals were fully converted into "COVID care," with abrupt cessation of essential services to non-COVID patients, who need regular care, for example, cancer, cardiac, trauma, obstetrics, as if they never existed. No one made alternative arrangements during peaks. Large civil hospitals shut their doors for non-COVID patients. In private hospitals, lack of space and supplies led to the closure of routine work. Many small-scale clinics and nursing homes shut due to staff or supply scarcity, fear, and failure to provide isolation facilities and comply with pandemic rules. Many patients must have perished due to nonavailability of "NON-COVID BEDS/Services," and no one has ever accounted for such sufferings and deaths. (2) Fear of contracting COVID infection at hospitals led many chronically ill patients to postpone or cancel their regular follow-ups and treatments, leading to flare-ups of latent diseases or diagnosis at a late stage of diseases. This has led to adverse outcomes. We do not have and may not ever have in the future, data on how many chemocycles were missed, coronary artery bypass graft surgery canceled, non-COVID illness remained undiagnosed, etc., leading to morbidity and mortalities. (3) Most neglected reason is misclassification or handling of COVID mimics between COVID and non-COVID facilities. This has led to long wait for first medical contact, delay in the workup, failure to evaluate differentials other than COVID or COVID-related illnesses (thought block), and prolonged turnaround time for outsource investigations (lack of public transport and lockdown restrictions). Pandemic protocols at times have added hurdles as senior clinicians diverted for COVID duties and non-COVID areas left unmanned or manned by junior scarce staff.

We all have many many experiences to share while caring for the patient in this COVID pandemic. In the midst of sea of COVID-19 pneumonia cases, diagnosis of ST-elevation myocardial infarction with pulmonary edema delayed for 48 hours. A pregnant female in her third trimester received high-dose steroids, just because she was COVID reverse transcriptase-polymerase chain reaction (RT-PCR) positive even if she is not hypoxic, not even symptomatic. Fracture neck femur surgery delayed just as surgeon wanted negative RT-PCR (which took 3 days at peak of (OVID wave) and spotless high-resolution computed tomography chest to be fit for surgery. Klebshiella pneumonia in chronic smoker required to stay next to corona-suspected patients as it took 3 days to prove the absence of corona infection. As it is well known, you can improve only what you can measure, we first need to measure
Department of Intensive Care, Sunshine Global Hospital, Surat, Gujarat, India

Corresponding Author: Anuj M Clerk, Department of Intensive Care, Sunshine Global Hospital, Surat, Gujarat, India, Phone: +91 7574851424, e-mail: anujmclerk@hotmail.com

How to cite this article: Clerk AM. Beware of Neglect of Non-COVID Patients in COVID Era. Indian J Crit Care Med 2021;25(8):837-838.

Source of support: Nil

Conflict of interest: None

this effect. We all have observed this in our units but the only way to study is to have unbiased vigilance by an adequately trained team with the sole goal of system improvement and not to create demoralizing, derogatory system remarks in social media.

Use of the definition of severe acute respiratory illness (SARI) as per the Guidelines of Ministry of Health, Government of India, ${ }^{1}$ has led to the classification of almost all patients of respiratory distress resulting in their admission to "COVID suspect ICU." When the gross majority of patients suffer from stereotyped clinical features during large-scale pandemic like this, it is usual for medicos and paramedicos to enter, in what I wish to call, a "Thought tunnel," where lateral thinking is reduced to near zero. Every patient is looked upon as having COVID infection and treated (at least initially) until the contrary is obvious or proven. This, at times, proves to be too late and misses the vital therapeutic window for best results. We all have witnessed this happening around us with resultant undue suffering of non-COVID patients.

The suffering of this group of patients is put to scrutiny in a unique observational cross-sectional study published in this issue of IJCCM by Arunachala et al. ${ }^{2}$ This study drew attention toward shortcomings in handling non-COVID patients arriving at major medical college hospitals during this pandemic. The authors presented a detailed description of delayed diagnosis, intervention, and treatment of non-COVID patients admitted to SARI ICU (intensive care unit) and its effects on morbidity and mortality. They studied 78 patients admitted to SARI ICU during the span of June to July 2020, in two 20 bedded multidisciplinary units of urban medical colleges hospitals in South India. If we start by looking at triaging of patients, compliance to the national advisory-based definition of SARI for triage was only $19.2 \%$, and only $26 \%$ of patients admitted to SARI ICU had respiratory symptoms. This happened when admission to SARI ICU was left to the discretion of physicians. This clearly depicts defensive decision-making under the pressure of statutory guidelines of local civic authorities as each cases, even suspected, is expected to be reported.

(c) Jaypee Brothers Medical Publishers. 2021 Open Access This article is distributed under the terms of the Creative Commons Attribution 4.0 International License (https://creativecommons.org/licenses/by-nc/4.0/), which permits unrestricted use, distribution, and non-commercial reproduction in any medium, provided you give appropriate credit to the original author(s) and the source, provide a link to the Creative Commons license, and indicate if changes were made. The Creative Commons Public Domain Dedication waiver (http://creativecommons.org/publicdomain/zero/1.0/) applies to the data made available in this article, unless otherwise stated. 
This defensive triaging has led to relatively lesser sick patients diverted to SARI ICU. This is reflected by: Only 33\% were on mechanical ventilator support, only $24 \%$ were on vasopressors, and the low mortality rate was $14.1 \%$ among this group. Nineteen percent of patients who left against medical advice have not only confounded outcome analysis but also reflect dissatisfaction of the patient or their relatives about the process of triaging and or handling (interventions and treatment) during these pandemic situations.

Internal and external validations are the best way to study the aspects of care without bias. Two independent observers in each unit were appointed for the purpose of the study to observe whether the incidence of endpoints of the study during pandemic situations is an admirable step for hardcore research in these taxing times. Though definitions of "delay" used were arbitrary but were a must for objective auditing. Delay in diagnosis (>24 hours for working diagnosis) was found in $17.9 \%$ and delay in intervention (as judged by Intensivist) noted in $23.1 \%$. Delay in cross-consultations ( $>4$ hours) was noted in $24.4 \%$ of patients in SARI ICU and delay in treatment ( $>24$ hours of admission to SARI ICU) in $12.8 \%$. Support from allied health services was affected in majority of cases and amounts be as high as $67.9 \%$ due to fear of visiting COVID areas of the hospital by healthcare workers of allied branches (physiotherapist, dietician, psychiatrist, counselors, etc.). Though none of these was found to be associated with outcome (mortality), high leaving against medical advice (LAMA) rate limits the fidelity of all these conclusions.

While conduction of research on a difficult subject like biased clinical decision making, delay in intervention, and treatment during staff-constrained times like pandemic is an admirable task, a small number of patients studied, high LAMA rates, only two government medical college hospitals with nonuniform staffing structure in centers studied, and lack of awareness among admitting physicians of the SARI definitions limit its widespread applicability and are also areas for improvement in future studies on the subject. However, this study definitely makes a point to prove that care of non-COVID patients was compromised in big way during COVID times.

The literature varies greatly between countries on this front. One report shows no change in pattern of Emergency Department (ED) visits, door to needle, and door to balloon times in one center in the first wave in Korea, ${ }^{3}$ while one from North Italy shows the contrary. In before and after analysis from Bologna metropolitan area in northern Italy, ${ }^{4}$ ED visits were significantly reduced. In the lockdown period, a rise was noted in overall out-of-hospital mortality (43.2\%). Cause-specific out-of-hospital mortality related to neoplasms (76.7\%), endocrine, nutritional, and metabolic (79.5\%) as well as cardiovascular (32.7\%) diseases was increased significantly.

The World Health Organization survey done in 155 countries (over 3 weeks in May 2020) showed marked disruption of services for noncommunicable diseases globally, but low-income countries affected the most. Fifty-three percent of countries have partially or completely disrupted services for hypertension treatment and $49 \%$ for the treatment for diabetes and its related complications. Forty-two percent of countries had a disruption in cancer care and $31 \%$ in cardiovascular emergencies. Rehabilitation services have been disrupted in over two-thirds (63\%) of countries during COVID pandemic. ${ }^{5}$
While data from India report significant disruption of routine services for non-COVID patients, ${ }^{6}$ a descriptive cross-sectional study based on Google-based questionnaire to caregivers of nonCOVID patients by Sarma et al. ${ }^{7}$ revealed 110 responses. And $56.4 \%$ of healthcare workers were disturbed by the lack of any concrete protocol for non-COVID patient management. Then, $68 \%$ expressed extreme stress in caring for non-COVID patients in COVID era and considered less staff availability, delay in discharging duties toward their patients, and increased workload are the drivers to their concerns. This burnout of healthcare providers contributes further to already stressed healthcare system.

Data generated in the current study by Arunachala et al., if collected from a large multicentric platform, can serve in planning for surge capacity and disaster management at the national level. Societies specializing in emergency medicine, anesthesiology, and intensive care can use such data in their guidelines on handling of such waves in the future. We need many more such system validation studies to reshape healthcare sector in our country, and for that attempts of authors need to appreciation. Till then "Beware of neglect of non-COVID patients" while planning for COVID care pathways.

\section{OrCID}

Anuj M Clerk @ https://orcid.org/0000-0001-8944-0077

\section{References}

1. Guidelines on clinical management of severe acute respiratory illness (SARI) in suspect/confirmed novel coronavirus ( $\mathrm{nCoV}$ ) cases [cited 2020, January 25]. Available from: https://main.mohfw.gov.in/sites/ default/files/Guidelines on Clinical management of severe acute respiratory illness.pdf.

2. Arunachala S, Venkatesh BT, Bhatt MT, PuranikA, Rao S, Ravindranath S. COVID-19 Pandemic: Impact on Admission, Diagnosis, and Treatment of Non-COVID-19 Patients Admitted to SARI ICU. Indian J Crit Care Med 2021; https://www.ijccm.org/doi/IJCCM/pdf/10.5005/ jp-journals-10071-23942.

3. Lee KD, Lee SB, Lim JK, Kang YM, Kim IB, Moon HJ, et al. Providing essential clinical care for non-COVID-19 patients in a Seoul metropolitan acute care hospital amidst ongoing treatment of COVID-19 patients. J Hosp Infect 2020;106(4):673-677. DOI: 10.1016/j. jhin.2020.09.031.

4. Santi L, Golinelli D, Tampieri A, Farina G, Greco M, Rosa S, et al. Non-COVID-19 patients in times of pandemic: emergency department visits, hospitalizations and cause-specific mortality in Northern Italy. PLoS One 2021;16(3):e0248995. DOI: 10.1371/journal. pone.0248995.

5. COVID-19 significantly impacts health services for noncommunicable diseases, WHO newsletter dated 1st June, 2020. Accessed from: https://www.who.int/news/item/01-06-2020-covid-19-significantlyimpacts-health-services-for-noncommunicable-diseases.

6. Babu N, Kohli P, Mishra C, Sen S, Arthur D, Chhablani D, et al. To evaluate the effect of COVID-19 pandemic and national lockdown on patient care at a tertiary-care ophthalmology institute. Indian J Ophthalmol 2020;68(8):1540-1544. DOI: 10.4103/ijo.IJO_1673_20. PMID: 32709770; PMCID: PMC7640832.

7. Sarma R, Vig S, Rathore P, Pushpam D, Mishra S, Gupta N, et al. Concerns of health care professionals managing non-COVID patients during the COVID-19 pandemic: a descriptive cross-sectional study. Indian J Palliat Care 2020;26(Suppl. S1):21-26. DOI: 10.4103/JJPC.IJPC_155_20. 